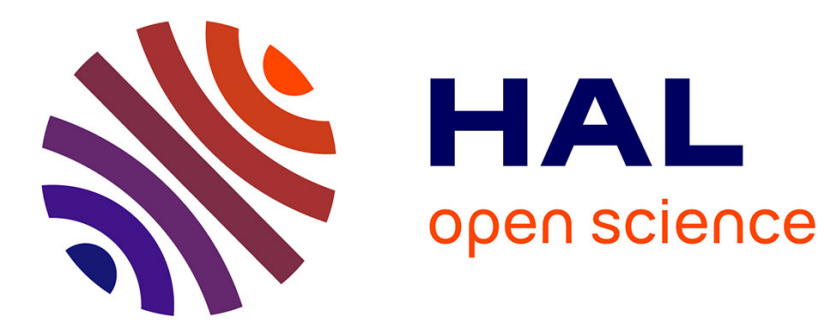

\title{
Interval analysis and dioid: application to robust controller design for timed event graphs
}

Mehdi Lhommeau, Laurent Hardouin, Bertrand Cottenceau, Luc Jaulin

\section{To cite this version:}

Mehdi Lhommeau, Laurent Hardouin, Bertrand Cottenceau, Luc Jaulin. Interval analysis and dioid: application to robust controller design for timed event graphs. Automatica, 2004, 40 (11), pp.19231930. 10.1016/j.automatica.2004.05.013 . hal-01113454

\section{HAL Id: hal-01113454 https://hal.science/hal-01113454}

Submitted on 5 Feb 2015

HAL is a multi-disciplinary open access archive for the deposit and dissemination of scientific research documents, whether they are published or not. The documents may come from teaching and research institutions in France or abroad, or from public or private research centers.
L'archive ouverte pluridisciplinaire HAL, est destinée au dépôt et à la diffusion de documents scientifiques de niveau recherche, publiés ou non, émanant des établissements d'enseignement et de recherche français ou étrangers, des laboratoires publics ou privés. 


\title{
Interval Analysis and Dioid : Application to Robust Controller Design for Timed Event Graphs *
}

\author{
Mehdi Lhommeau, Laurent Hardouin, Bertrand Cottenceau, \\ Luc Jaulin \\ Laboratoire d'Ingénierie des Systèmes Automatisés \\ 62, avenue Notre Dame du Lac, 49000 Angers (France)
}

\begin{abstract}
This paper deals with feedback controller synthesis for timed event graphs in dioids, where the number of initial tokens and time delays are only known to belong to intervals. We discuss here the existence and the computation of a robust controller set for uncertain systems that can be described by parametric models, the unknown parameters of which are assumed to vary between known bounds. Each controller is computed in order to guarantee that the closed-loop system behavior is greater than the lower bound of a reference model set and is lower than the upper bound of this set. The synthesis presented here is mainly based on dioid, interval analysis and residuation theory.
\end{abstract}

Key words: Discrete-event systems ; Timed event graphs ; Dioid ; Interval analysis ; Residuation theory ; Feedback synthesis

\section{Introduction}

Discrete Event Systems (DES) appear in many applications in manufacturing systems [1], computer and communication systems [5] and are often described

ऋ This paper was not presented at any IFAC meeting. Corresponding author M. Lhommeau. Tel. +33(0)2.41.36.57. Fax +XXXIX-VI-mmmxxv.

Email addresses: 1hommeau@istia.univ-angers.fr (Mehdi Lhommeau), hardouin@istia. univ-angers. fr (Laurent Hardouin), cottence@istia.univ-angers.fr (Bertrand Cottenceau), jaulin@univ-angers.fr (Luc Jaulin).

Preprint submitted to Elsevier Science 20 June 2003 
by the Petri Net formalism. Timed-Event Graphs (TEG) are Timed Petri Nets in which all places have single upstream and single downstream transitions and appropriately model DES characterized by delay and synchronization phenomena. TEG can be described by linear equations in the dioid algebra $[2,7]$ and this fact has permitted many important achievements on the control of DES modelled by TEG $[7,8,17,15]$. TEG control problems are usually stated in a Just-in-time context. The design goal is to achieve some performance while minimizing internal stocks. In $[2,17]$ an optimal open-loop control law is given. In [8] linear closed-loop controllers synthesis are given in a model matching objective, i.e., a given reference model describes the desired performance limits, then the goal is to compute a feedback controller in order to obtain a closed-loop behavior as close as possible to the reference model and to delay as much as possible the inputs in the system.

This paper aims at designing robust feedback controller when the system includes some parametric uncertainties which can be described by intervals. Intervals allow to describe TEG with number of tokens and/or time delays, which are assumed to vary between known bounds. Assuming that there exists a lower and an upper bound to a specification set, the synthesis yields a controller set which guarantees that the closed loop system behavior is both greater than the lower bound of the specification set and lower than the upper bound of this same set ${ }^{1}$. Controller synthesis is obtained by considering residuation theory which allows the inversion of mapping defined over ordered sets, and interval analysis which is known to be efficient to characterize set of robust controllers in a guaranteed way [12].

The next Section introduces algebraic tools on dioid and residuation theory. Section 3 and 4 give the main results, dioid of interval $\mathrm{I}(\mathcal{D})$ is constructed and mapping inversion over $\mathrm{I}(\mathcal{D})$ is addressed. The problem of robust controller synthesis when the system includes interval parametric uncertainties is stated and solved in Sections 5 and 6. Section 7 presents an illustrative example.

\section{Dioids and Residuation}

A dioid $\mathcal{D}$ is a set endowed with two internal operations denoted $\oplus$ (addition) and $\otimes$ (multiplication), both associative and both having neutral elements denoted $\varepsilon$ and $e$ respectively, such that $\oplus$ is also commutative and idempotent (i.e. $a \oplus a=a$ ). The $\otimes$ operation is distributive with respect to $\oplus$, and $\varepsilon$ is absorbing for the product (i.e. $\varepsilon \otimes a=a \otimes \varepsilon=\varepsilon, \forall a$ ). When $\otimes$ is commutative, the dioid is said to be commutative. The symbol $\otimes$ is often omitted.

Dioids can be endowed with a natural order : $a \succeq b$ iff $a=a \oplus b$. Then

1 From TEG point of view it is a set of robust controller which ensures that the controlled system is both slower than a reference model (described as a TEG) and faster than another one. 
they become sup-semilattices and $a \oplus b$ is the least upper bound of $a$ and $b$. A dioid is complete if sums of infinite number of terms are always defined, and if multiplication distributes over infinite sums too. In particular, the sum of all elements of the dioid is defined and denoted $T$ (for 'top'). A complete dioid (sup-semilattice) becomes a lattice by constructing the greatest lower bound of $a$ and $b$, denoted $a \wedge b$, as the least upper bound of the (nonempty) subset of all elements which are less than $a$ and $b$ (see $[2, \S 4]$ ).

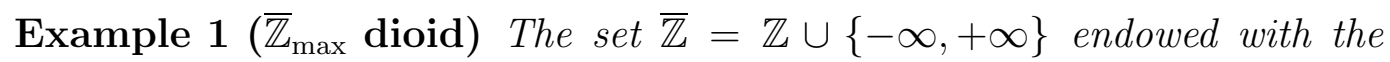
max operator as sum and the classical sum + as product is a complete dioid, usually denoted by $\overline{\mathbb{Z}}_{\max }$, of which $\varepsilon=-\infty$ and $e=0$.

Definition 2 (Subdioid) A subset $\mathcal{C}$ of a dioid is called a subdioid of $\mathcal{D}$ if

- $\varepsilon \in \mathcal{C}$ and $e \in \mathcal{C}$;

- $\mathcal{C}$ is closed for $\oplus$ and $\otimes$.

The second statement means, $\forall a, b \in \mathcal{C}, a \oplus b \in \mathcal{C}$ and $a \otimes b \in \mathcal{C}$.

Remark 3 Obviously a subdioid of a complete dioid may be not complete.

Theorem 4 Over a complete dioid $\mathcal{D}$, the implicit equation $x=a x \oplus b$ admits $x=a^{*} b$ as least solution, where $a^{*}=\bigoplus_{i \in \mathbb{N}} a^{i}$ (Kleene star operator) with $a^{0}=e$.

The Kleene star operator, over a complete dioid $\mathcal{D}$, will be sometimes represented by the following mapping $\mathcal{K}: \mathcal{D} \rightarrow \mathcal{D}, x \mapsto x^{*}$.

Theorem 5 ([8]) Let $\mathcal{D}$ be a complete dioid and $a, b \in \mathcal{D}$.

$$
\begin{aligned}
a(b a)^{*} & =(a b)^{*} a, \\
\left(a^{*}\right)^{*} & =a^{*}
\end{aligned}
$$

\subsection{Residuation theory}

The residuation theory provides, under some assumptions, optimal solutions to inequalities such as $f(x) \preceq b$ where $f$ is an isotone mapping ( $f$ s.t. $a \preceq$ $b \Rightarrow f(a) \preceq f(b))$ defined over ordered sets. Some theoretical results are summarized below. Basic references are [4] and [2, §4.4.2].

Definition 6 (Residual and residuated mapping) An isotone mapping $f$ : $\mathcal{D} \rightarrow \mathcal{E}$, where $\mathcal{D}$ and $\mathcal{E}$ are ordered sets, is a residuated mapping if for all $y \in \mathcal{E}$, the least upper bound of the subset $\{x \mid f(x) \preceq y\}$ exists and belongs to this subset. It is then denoted $f^{\sharp}(y)$. Mapping $f^{\sharp}$ is called the residual of $f$. 
When $f$ is residuated, $f^{\sharp}$ is the unique isotone mapping such that

$$
f \circ f^{\sharp} \preceq \mathrm{Id}_{\mathcal{E}} \quad \text { and } f^{\sharp} \circ f \succeq \mathrm{Id}_{\mathcal{D}},
$$

where Id is the identity mapping respectively on $\mathcal{D}$ and $\mathcal{E}$.

Property 7 Let $f: \mathcal{D} \rightarrow \mathcal{E}$ be a residuated mapping, then

$$
y \in f(\mathcal{D}) \Leftrightarrow f\left(f^{\sharp}(y)\right)=y .
$$

Property 8 ([2, Th. 4.56]) If $h: \mathcal{D} \rightarrow \mathcal{C}$ and $f: \mathcal{C} \rightarrow \mathcal{B}$ are residuated mapping, then $f \circ h$ is also residuated and

$$
(f \circ h)^{\sharp}=h^{\sharp} \circ f^{\sharp} .
$$

Theorem 9 ([2, §4.4.2]) Consider the mapping $f: \mathcal{E} \rightarrow \mathcal{F}$ where $\mathcal{E}$ and $\mathcal{F}$ are complete dioids of which the bottom elements are, respectively, denoted by $\varepsilon_{\mathcal{E}}$ and $\varepsilon_{\mathcal{F}}$. Then, $f$ is residuated iff $f\left(\varepsilon_{\mathcal{E}}\right)=\varepsilon_{\mathcal{F}}$ and $f\left(\bigoplus_{x \in \mathcal{G}} x\right)=\bigoplus_{x \in \mathcal{G}} f(x)$ for each $\mathcal{G} \subseteq \mathcal{E}$ (i.e $f$ is lower-semicontinuous abbreviated l.s.c.).

Corollary 10 The mappings $L_{a}: x \mapsto$ ax and $R_{a}: x \mapsto x a$ defined over $a$ complete dioid $\mathcal{D}$ are both residuated. ${ }^{2}$ Their residuals are usually denoted, respectively, $L_{a}^{\sharp}(x)=a ф x$ and $R_{a}^{\sharp}(x)=x \phi a$ in $(\max ,+)$ literature. $^{3}$

Theorem 11 ([2, §4.4.4]) The mappings $x \mapsto a ф x$ and $x \mapsto x \phi a$ verify the following properties :

$$
\begin{aligned}
& (a b) \phi x=b \phi(a \phi x) \quad x \phi(b a)=(x \phi a) \phi b, \\
& a^{*} x=a^{*} \phi\left(a^{*} x\right) \quad x a^{*}=\left(x a^{*}\right) \phi a^{*}, \\
& a \phi(x \wedge y)=a \phi x \wedge a ф y \quad(x \wedge y) \phi a=x \phi a \wedge y \phi a \text {. }
\end{aligned}
$$

Theorem 12 ([18]) Let $\mathcal{D}$ be a complete dioid and $A \in \mathcal{D}^{p \times n}$ be a matrix with entries in $\mathcal{D}$. Then, $A \nmid A$ is a matrix in $\mathcal{D}^{n \times n}$ which verifies

$$
A ф A=(A ф A)^{*}
$$

\subsection{Mapping restriction}

In this subsection, the problem of mapping restriction and its connection with the residuation theory is addressed. In particular the Kleene star mapping,

2 This property concerns as well a matrix dioid product, for instance $X \mapsto A X$ where $A, X \in \mathcal{D}^{n \times n}$. See [2] for the computation of $A \phi B$ and $B \phi A$.

$3 a ф b$ is the greatest solution of $a x \preceq b$. 
becomes residuated as soon as its codomain is restricted to its image.

Definition 13 (Restricted mapping) Let $f: \mathcal{E} \rightarrow \mathcal{F}$ be a mapping and $\mathcal{A} \subseteq \mathcal{E}$. We will denote $f_{\mid \mathcal{A}}: \mathcal{A} \rightarrow \mathcal{F}$ the mapping defined by $f_{\mid \mathcal{A}}=f \circ \operatorname{ld}_{\mid \mathcal{A}}$ where $\operatorname{ld}_{\mid \mathcal{A}}: \mathcal{A} \rightarrow \mathcal{E}, x \mapsto x$ is the canonical injection. Identically, let $\mathcal{B} \subseteq \mathcal{F}$ with $\operatorname{Im} f \subseteq \mathcal{B}$. Mapping ${ }_{\mathcal{B} \mid} f: \mathcal{E} \rightarrow \mathcal{B}$ is defined by $f=\operatorname{ld}_{\mid \mathcal{B}} \circ{ }_{\mathcal{B} \mid} f$, where $\operatorname{Id}_{\mid \mathcal{B}}: \mathcal{B} \rightarrow \mathcal{F}, x \mapsto x$ is the canonical injection.

Proposition 14 (Canonical injection) Let $\operatorname{ld}_{\mid \mathcal{D}_{\text {sub }}}: \mathcal{D}_{\text {sub }} \rightarrow \mathcal{D}$ be the canonical injection from a complete subdioid into a complete dioid. The injection $\mathrm{Id}_{\mid \mathcal{D}_{\text {sub }}}$ is residuated and its residual will be denoted

$$
\left(\operatorname{ld}_{\mid \mathcal{D}_{\text {sub }}}\right)^{\sharp}=\operatorname{Pr}_{\text {sub }} \text {. }
$$

Remark 15 (Constrained Residuation) The residuation theory provides the greatest solution of $f(x) \preceq b$, where $f: \mathcal{D} \rightarrow \mathcal{E}$ is an isotone mapping. The constrained residuation means that we look for the 'approximate' solution not in whole $\mathcal{D}$ but only in a subdioid $\mathcal{D}_{\text {sub }}$ of $\mathcal{D}$.

Theorem $16([6, \S 1.3])$ Let $\operatorname{ld}_{\mid \mathcal{D}_{\text {sub }}}$ the canonical injection from $\mathcal{D}_{\text {sub }}$ to $\mathcal{D}$. Solving $f(x) \preceq b$ amounts to solving

$$
f \circ \operatorname{Id}_{\mid \mathcal{D}_{\text {sub }}}(x) \preceq b
$$

for the greatest solution in $\mathcal{D}_{\text {sub. If }} \mathcal{D}_{\text {sub }}$ is a complete subdioid, then $\operatorname{ld}_{\mid \mathcal{D}_{\text {sub }}}$ is residuated and the answer is

$$
\left(f \circ \operatorname{Id}_{\mid \mathcal{D}_{\text {sub }}}\right)^{\sharp}(b)=\left(\operatorname{Id}_{\mid \mathcal{D}_{\text {sub }}}\right)^{\sharp} \circ f^{\sharp}(b) \quad \text { (thanks to Property 8). }
$$

Definition 17 (Closure mapping) An isotone mapping $f: \mathcal{E} \rightarrow \mathcal{E}$ defined on an ordered set $\mathcal{E}$ is a closure mapping if $f \succeq \mathrm{Id}_{\mathcal{E}}$ and $f \circ f=f$.

Proposition $18([8])$ Let $f: \mathcal{E} \rightarrow \mathcal{E}$ be a closure mapping. A closure mapping restricted to its image $\operatorname{Im} f \mid f$ is a residuated mapping whose residual is the canonical injection $\operatorname{Id}_{\operatorname{Im} f}: \operatorname{Im} f \rightarrow \mathcal{E}, x \mapsto x$.

Corollary 19 The mapping ${ }_{\operatorname{Im} \mathcal{K} \mid} \mathcal{K}$ is a residuated mapping whose residual is $(\operatorname{Im} \mathcal{K} \mid \mathcal{K})^{\sharp}=\operatorname{Id}_{\mid \operatorname{Im} \mathcal{K}}$.

This means that $x=a^{*}$ is the greatest solution to inequality $x^{*} \preceq a^{*}$. Actually, the greatest solution achieves equality.

Proposition 20 Let $M_{a}: x \mapsto(a x)^{*}$ a be a mapping defined over a complete dioid. Consider $g \in \mathcal{D}$ and $d \in \mathcal{D}$. Let us consider the following sets :

$\overline{4}$ These notations are borrowed from classical linear system theory see [20]. 


$$
\begin{aligned}
& \mathcal{G}_{1}=\left\{g \mid \exists d \text { s.t. } g=d^{*} a\right\}, \\
& \mathcal{G}_{2}=\left\{g \mid \exists d \text { s.t. } g=a d^{*}\right\} .
\end{aligned}
$$

$\operatorname{Im} M_{a} \subseteq\left(\mathcal{G}_{1} \cap \mathcal{G}_{2}\right)$ and the mappings ${ }_{\mathcal{G}_{1} \mid} M_{a}$ and ${ }_{\mathcal{G}_{1} \mid} M_{a}$ are both residuated. Their residuals are such that $\left(\mathcal{G}_{1} \mid M_{a}\right)^{\sharp}(x)=\left(\mathcal{G}_{2} \mid M_{a}\right)^{\sharp}(x)=a ф x \phi a$.

PROOF. Equation (1) leads to $(a x)^{*} a=a(x a)^{*}$, then by choosing $d=a x$ or $d=x a$, it comes $\operatorname{Im} M_{a} \subseteq\left(\mathcal{G}_{1} \cap \mathcal{G}_{2}\right)$. According to Definition 6 , we remark that the following assertions are equivalent :

- $\mathcal{G}_{1} \mid M_{a}$ is residuated.

- $\forall d \in \mathcal{D},(a x)^{*} a \preceq d^{*} a$ admits a greatest solution.

So, we can concentrate on the second point. Since the mapping $L_{a}$ is residuated (cf. Corollary 10) and according to (1), we have

$$
(a x)^{*} a=a(x a)^{*} \preceq d^{*} a \Leftrightarrow(x a)^{*} \preceq a \phi\left(d^{*} a\right) .
$$

According to (6) and (5), we can rewrite $a \oint\left(d^{*} a\right)=a \oint\left(d^{*} \phi\left(d^{*} a\right)\right)=\left(d^{*} a\right) \oint\left(d^{*} a\right)$. According to (8), this last expression shows that $a \nmid\left(d^{*} a\right)$ belongs to the image of $\mathcal{K}$. Since ${ }_{\operatorname{Im} \mathcal{K} \mid} \mathcal{K}$ is residuated (cf. Corollary 19), there is also the following equivalence:

$$
(x a)^{*} \preceq a \phi\left(d^{*} a\right) \Leftrightarrow x a \preceq a \phi\left(d^{*} a\right) .
$$

Finally, since $R_{a}$ is residuated too (cf. Corollary 10), we verify that $x=$ $a \oint\left(d^{*} a\right) \phi a$ is the greatest solution of $(a x)^{*} a \preceq d^{*} a, \forall d \in \mathcal{D}$. That amounts to saying that $\mathcal{G}_{1} \mid M_{a}$ is residuated. We would show that $\mathcal{G}_{2} \mid M_{a}$ is residuated with analog steps.

Corollary 21 If $g \in \operatorname{Im} M_{a}$, then $x=a ф g \phi a$ is the greatest solution to the equation $(a x)^{*} a=g$.

PROOF. First $\operatorname{Im} M_{a} \subseteq\left(\mathcal{G}_{1} \cap \mathcal{G}_{2}\right)$, thus ${\operatorname{Im} M_{a} \mid}_{a} M_{a}$ is residuated. Furthermore, $\forall y \in \operatorname{Im} M_{a}, M_{a}(x)=y$ admits a solution, i.e., $\operatorname{Im} M_{a} \mid M_{a}$ is surjective, then $\left(\operatorname{Im} M_{a} \mid M_{a}\right)^{\sharp}$ provides the greatest solution (see Property 7 ). 


\section{$3 \quad$ Dioid $\mathcal{C}(\mathcal{D})$}

Consider the set of pairs $\left(x^{\prime}, x^{\prime \prime}\right)$ with $x^{\prime} \in \mathcal{D}$ and $x^{\prime \prime} \in \mathcal{D}$ endowed with two coordinate-wise algebraic operations :

$$
\begin{aligned}
& \left(x^{\prime}, x^{\prime \prime}\right) \tilde{\oplus}\left(y^{\prime}, y^{\prime \prime}\right)=\left(x^{\prime} \oplus y^{\prime}, x^{\prime \prime} \oplus y^{\prime \prime}\right), \\
& \left(x^{\prime}, x^{\prime \prime}\right) \tilde{\otimes}\left(y^{\prime}, y^{\prime \prime}\right)=\left(x^{\prime} \otimes y^{\prime}, x^{\prime \prime} \otimes y^{\prime \prime}\right),
\end{aligned}
$$

This set is a dioid denoted $\mathcal{C}(\mathcal{D})$ with $(\varepsilon, \varepsilon)$ as the zero element and $(e, e)$ as the identity element.

Remark 22 The operation $\tilde{\oplus}$ generates the corresponding canonical partial order $\preceq_{\mathcal{C}}$ in $\mathcal{C}(\mathcal{D})$ :

$\left(x^{\prime}, x^{\prime \prime}\right) \widetilde{\oplus}\left(y^{\prime}, y^{\prime \prime}\right)=\left(y^{\prime}, y^{\prime \prime}\right) \Leftrightarrow\left(x^{\prime}, x^{\prime \prime}\right) \preceq_{\mathcal{C}}\left(y^{\prime}, y^{\prime \prime}\right) \Leftrightarrow x^{\prime} \preceq_{\mathcal{D}} y^{\prime}$ and $x^{\prime \prime} \preceq_{\mathcal{D}} y^{\prime \prime}$ where $\preceq_{\mathcal{D}}$ is the order relation in $\mathcal{D}$.

Proposition 23 ([13]) If the dioid $\mathcal{D}$ is complete, then the dioid $\mathcal{C}(\mathcal{D})$ is complete.

Proposition 24 An isotone mapping $f$ defined over $\mathcal{D}$ has a natural extension in $\mathcal{C}(\mathcal{D}), f\left(x^{\prime}, x^{\prime \prime}\right)=\left(f\left(x^{\prime}\right), f\left(x^{\prime \prime}\right)\right)$.

Example 25 The Kleene star operation in $\mathcal{C}(\mathcal{D})$ is defined by $\mathcal{K}\left(x^{\prime}, x^{\prime \prime}\right)=$ $\left(\mathcal{K}\left(x^{\prime}\right), \mathcal{K}\left(x^{\prime \prime}\right)\right)=\left(x^{\prime *}, x^{\prime \prime *}\right)$.

Now we consider the following mappings over $\mathcal{C}(\mathcal{D})$ :

$$
\begin{array}{lr}
L_{\left(a^{\prime}, a^{\prime \prime}\right)}:\left(x^{\prime}, x^{\prime \prime}\right) \mapsto\left(a^{\prime}, a^{\prime \prime}\right) \widetilde{\otimes}\left(x^{\prime}, x^{\prime \prime}\right) & \text { (left multiplication by } \left.\left(a^{\prime}, a^{\prime \prime}\right)\right) ; \\
R_{\left(a^{\prime}, a^{\prime \prime}\right)}:\left(x^{\prime}, x^{\prime \prime}\right) \mapsto\left(x^{\prime}, x^{\prime \prime}\right) \widetilde{\otimes}\left(a^{\prime}, a^{\prime \prime}\right) & \text { (right multiplication by } \left.\left(a^{\prime}, a^{\prime \prime}\right)\right) .
\end{array}
$$

Proposition 26 The mappings $L_{\left(a^{\prime}, a^{\prime \prime}\right)}$ and $R_{\left(a^{\prime}, a^{\prime \prime}\right)}$ defined over $\mathcal{C}(\mathcal{D})$ are both residuated. Their residuals are equal to $L_{\left(a^{\prime}, a^{\prime \prime}\right)}^{\sharp}\left(b^{\prime}, b^{\prime \prime}\right)=\left(a^{\prime}, a^{\prime \prime}\right) \phi\left(b^{\prime}, b^{\prime \prime}\right)=$ $\left(a^{\prime} \phi b^{\prime}, a^{\prime \prime} \phi b^{\prime \prime}\right)$ and $R_{\left(a^{\prime}, a^{\prime \prime}\right)}^{\sharp}\left(b^{\prime}, b^{\prime \prime}\right)=\left(b^{\prime}, b^{\prime \prime}\right) \phi\left(a^{\prime}, a^{\prime \prime}\right)=\left(b^{\prime} \phi a^{\prime}, b^{\prime \prime} \phi a^{\prime \prime}\right)$.

PROOF. Observe that $L_{\left(a^{\prime}, a^{\prime \prime}\right)}\left(\bigoplus_{\left(x^{\prime}, x^{\prime \prime}\right) \in X}\left(x^{\prime}, x^{\prime \prime}\right)\right)=\bigoplus_{\left(x^{\prime}, x^{\prime \prime}\right) \in X} L_{\left(a^{\prime}, a^{\prime \prime}\right)}\left(x^{\prime}, x^{\prime \prime}\right)$, (for every subset $X$ of $\mathcal{C}(\mathcal{D})$ ), moreover $L_{\left(a^{\prime}, a^{\prime \prime}\right)}(\varepsilon, \varepsilon)=\left(a^{\prime} \varepsilon, a^{\prime \prime} \varepsilon\right)=(\varepsilon, \varepsilon)$. Then $L_{\left(a^{\prime}, a^{\prime \prime}\right)}$ is residuated (thanks to Theorem 9). Therefore, we have to find, for given $\left(b^{\prime}, b^{\prime \prime}\right)$ and $\left(a^{\prime}, a^{\prime \prime}\right)$, the greatest solution $\left(x^{\prime}, x^{\prime \prime}\right)$ for inequality $\left(a^{\prime}, a^{\prime \prime}\right) \widetilde{\otimes}\left(x^{\prime}, x^{\prime \prime}\right) \preceq_{\mathcal{C}}\left(b^{\prime}, b^{\prime \prime}\right) \Leftrightarrow\left(a^{\prime} \otimes x^{\prime}, a^{\prime \prime} \otimes x^{\prime \prime}\right) \preceq_{\mathcal{C}}\left(b^{\prime}, b^{\prime \prime}\right)$, moreover according to Remark 22 on the order relation induced by $\oplus$ on $\mathcal{C}(\mathcal{D})$ we have,

$$
a^{\prime} \otimes x^{\prime} \preceq_{\mathcal{D}} b^{\prime} \text { and } a^{\prime \prime} \otimes x^{\prime \prime} \preceq_{\mathcal{D}} b^{\prime \prime}
$$


Since the mappings $x^{\prime} \mapsto a^{\prime} \otimes x^{\prime}$ and $x^{\prime \prime} \mapsto a^{\prime \prime} \otimes x^{\prime \prime}$ are residuated over $\mathcal{D}$ (cf. Corollary 10), we have $x^{\prime} \preceq_{\mathcal{D}} a^{\prime} \phi b^{\prime}$ and $x^{\prime \prime} \preceq_{\mathcal{D}} a^{\prime \prime} \phi b^{\prime \prime}$. Then, we obtain $L_{\left(a^{\prime}, a^{\prime \prime}\right)}^{\sharp}\left(b^{\prime}, b^{\prime \prime}\right)=\left(a^{\prime} \phi b^{\prime}, a^{\prime \prime} \phi b^{\prime \prime}\right)$.

\section{Dioid and Interval Mathematics}

Interval mathematics was pioneered by Ramon E. Moore as a tool for bounding rounding and truncation errors in computer programs. Since then, interval mathematics had been developed into a general methodology for investigating numerical uncertainty in numerous problems and algorithms, and is a powerful numerical tool for calculating guaranteed bounds on functions using computers.

In [13] the problem of interval mathematics in dioids is addressed. The authors give a weak interval extensions of dioids and show that idempotent interval mathematics appears to be remarkably simpler than its traditional analog. For example, in the traditional interval arithmetic, multiplication of intervals is not distributive with respect to addition of intervals, while idempotent interval arithmetic keeps this distributivity. Below, we state that residuated theory has a natural extension in dioid of intervals.

\subsection{Interval arithmetic in dioid}

A (closed) interval in dioid $\mathcal{D}$ is a set of the form $\mathbf{x}=[\underline{x}, \bar{x}]=\{t \in \mathcal{D} \mid \underline{x} \preceq t \preceq$ $\bar{x}$, where $(\underline{x}, \bar{x}) \in \mathcal{C}(\mathcal{D}), \underline{x}$ (respectively, $\bar{x}$ ) is said to be lower (respectively, upper) bound of the interval $\mathbf{x}$. In $[13]$ the authors define dioid $\mathrm{I}(\mathcal{D})$ endowed with two coordinate-wise algebraic operations :

$$
\begin{aligned}
& \mathbf{x} \bar{\oplus} \mathbf{y}=[\underline{x} \oplus \underline{y}, \bar{x} \oplus \bar{y}] \\
& \mathbf{x} \bar{\otimes} \mathbf{y}=[\underline{x} \otimes \underline{y}, \bar{x} \otimes \bar{y}]
\end{aligned}
$$

where the interval $\varepsilon=[\varepsilon, \varepsilon]$ (respectively, $\mathbf{e}=[e, e]$ ) is zero (respectively, unit) element of $\mathrm{I}(\mathcal{D})$.

Since $\underline{x} \oplus \underline{y} \preceq \bar{x} \oplus \bar{y}$ and $\underline{x} \otimes \underline{y} \preceq \bar{x} \otimes \bar{y}$ whenever $\underline{x} \preceq \bar{x}$ and $\underline{y} \preceq \bar{y}$, then $\mathrm{I}(\mathcal{D})$ is closed with respect to the operations $\bar{\oplus}, \bar{\otimes}$, furthermore zero element and unit element of $\mathcal{C}(\mathcal{D})$ are in $\mathrm{I}(\mathcal{D})$; hence $\mathrm{I}(\mathcal{D})$ is a subdioid of $\mathcal{C}(\mathcal{D})$ (see Definition 2$)$. I $(\mathcal{D})$ may be not complete, but can be completed by considering the following definition (see [13], [10] and [3] for an ordered set topology introduction). 
Definition 27 Let $\left\{\mathbf{x}_{\alpha}\right\}$ be an infinite subset of $\mathrm{I}(\mathcal{D})$, the infinite sum of elements of this subset is:

$$
\overline{\bigoplus_{\alpha}} \mathbf{x}_{\alpha}=\left[\bigoplus_{\alpha} \underline{x}_{\alpha}, \bigoplus_{\alpha} \bar{x}_{\alpha}\right]
$$

Remark 28 Note that if $\mathbf{x}$ and $\mathbf{y}$ are intervals in $\mathrm{I}(\mathcal{D})$, then $\mathbf{x} \subset \mathbf{y}$ iff $\underline{y} \preceq$ $\underline{x} \preceq \bar{x} \preceq \bar{y}$. In particular, $\mathbf{x}=\mathbf{y}$ iff $\underline{x}=\underline{y}$ and $\bar{x}=\bar{y}$.

Remark 29 An interval for which $\underline{x}=\bar{x}$ is called degenerate. Degenerate intervals allow to represent numbers without uncertainty. In this case we identify $\mathbf{x}$ with its element by writing $\mathbf{x} \equiv x$.

\subsection{Residuation of interval linear inequations}

Proposition 30 The canonical injection $\operatorname{ld}_{\mid \mathrm{I}(\mathcal{D})}: \mathrm{I}(\mathcal{D}) \rightarrow \mathcal{C}(\mathcal{D})$ is residuated. Its residual will be denoted $\operatorname{Pr}_{\mathrm{I}}$, i.e.

$$
\left(\operatorname{ld}_{\mid \mathrm{I}(\mathcal{D})}\right)^{\sharp}=\operatorname{Pr}_{\mathrm{I}}
$$

PROOF. It is a direct application of Theorem 16 , since $\mathrm{I}(\mathcal{D})$ is a subdioid of $\mathcal{C}(\mathcal{D})$. The practical computation of $\operatorname{Pr}_{\mathrm{I}}$ is obtained as follows. Let $\left(x^{\prime}, x^{\prime \prime}\right) \in$ $\mathcal{C}(\mathcal{D}), \operatorname{Pr}_{\mathrm{I}}\left(x^{\prime}, x^{\prime \prime}\right)=[\underline{x}, \bar{x}]=\left[x^{\prime} \wedge x^{\prime \prime}, x^{\prime \prime}\right]$, which is the greatest interval such that:

$$
\underline{x} \preceq x^{\prime}, \bar{x} \preceq x^{\prime \prime} \text { and } \underline{x} \preceq \bar{x} \text {. }
$$

Proposition 31 Mapping $L_{\mathbf{a}}: \mathrm{I}(\mathcal{D}) \rightarrow \mathrm{I}(\mathcal{D}), \mathbf{x} \mapsto \mathbf{a} \bar{\otimes} \mathbf{x}$ is residuated. Its residual is equal to $L_{\mathbf{a}}^{\sharp}(\mathbf{b})=\mathbf{a} \phi \mathbf{b}=[\underline{a} \oint \underline{b} \wedge \bar{a} \phi \bar{b}, \bar{a} \phi \bar{b}]$.

PROOF. Observe that $L_{\mathbf{a}}$ is l.s.c., i.e. $L_{\mathbf{a}}\left(\bar{\oplus}_{\mathbf{x} \in \mathbf{x}} \mathbf{x}\right)=\bar{\oplus}_{\mathbf{x} \in \mathbf{x}} L_{\mathbf{a}}(\mathbf{x})$, (for every subset $\mathbf{X}$ of $\mathrm{I}(\mathcal{D}))$, moreover $L_{\mathbf{a}}(\varepsilon)=L_{\mathbf{a}}([\varepsilon, \varepsilon])=[\underline{a} \varepsilon, \bar{a} \varepsilon]=[\varepsilon, \varepsilon]=\varepsilon$. Then $L_{\mathbf{a}}$ is residuated (see Theorem 9 ). By considering result about constrained residuation (Theorem 16) and since the canonical injection is residuated (Proposition 30), we have

$$
\left(L_{\left(a^{\prime}, a^{\prime \prime}\right)} \circ \operatorname{Id}_{\mid \mathrm{I}(\mathcal{D})}\right)^{\sharp}=\left(\operatorname{Id}_{\mid \mathrm{I}(\mathcal{D})}\right)^{\sharp} \circ\left(L_{\left(a^{\prime}, a^{\prime \prime}\right)}\right)^{\sharp}=\operatorname{Pr}_{\mathrm{I}} \circ\left(L_{\left(a^{\prime}, a^{\prime \prime}\right)}\right)^{\sharp} .
$$

Then by considering $\mathbf{b} \in \mathrm{I}(\mathcal{D}) \subset \mathcal{C}(\mathcal{D})$ the greatest solution in $\mathrm{I}(\mathcal{D})$ of $\mathbf{A} \bar{\otimes}$ $\mathbf{x} \preceq \mathbf{b}$ is $\mathbf{x}=\mathbf{A} \phi \mathbf{b}=[\underline{x}, \bar{x}]=[\underline{A} \phi \underline{b} \wedge \bar{A} \phi \bar{b}, \bar{A} \phi \bar{b}]$. 
Remark 32 We would show in the same manner that mapping $R_{\mathbf{a}}: \mathrm{I}(\mathcal{D}) \rightarrow$ $\mathrm{I}(\mathcal{D}), \mathbf{x} \mapsto \mathbf{x} \bar{\otimes} \mathbf{a}$ is residuated .

Remark 33 We have seen that it is possible to extend the Kleene star operator over $\mathrm{I}(\mathcal{D})$ (see Example 25). Then $\operatorname{ImK} \mid \mathbf{K}$ is also a residuated mapping (see Corollary 19) whose residual is $(\operatorname{ImK} \mid \mathbf{K})^{\sharp}=\operatorname{Id}_{\mid \operatorname{ImK} \mathbf{K}}$. This means that $\mathbf{x}=\mathbf{a}^{*}$ is the greatest solution to inequality $\mathbf{x}^{*}=\left[\underline{x}^{*}, \bar{x}^{*}\right] \preceq \mathbf{a}^{*}=\left[\underline{a}^{*}, \bar{a}^{*}\right]$.

\section{Interval arithmetic and Timed Event Graphs}

It is well known that the behavior of a TEG can be expressed by linear state equations over some dioids, e.g., over dioid of formal power series with coefficients in $\overline{\mathbb{Z}}_{\max }$ and exponents in $\mathbb{Z}$ namely $\overline{\mathbb{Z}}_{\max } \llbracket \gamma \rrbracket$.

$$
\begin{aligned}
& X=A X \oplus B U \\
& Y=C X
\end{aligned}
$$

Where $X \in\left(\overline{\mathbb{Z}}_{\max } \llbracket \gamma \rrbracket\right)^{n}$ represents the internal transitions behavior, $U \in$ $\left(\overline{\mathbb{Z}}_{\max } \llbracket \gamma \rrbracket\right)^{p}$ represents the input transitions behavior, and $Y \in\left(\overline{\mathbb{Z}}_{\max } \llbracket \gamma \rrbracket\right)^{q}$ represents the output transitions behavior, and $A \in\left(\overline{\mathbb{Z}}_{\max } \llbracket \gamma \rrbracket\right)^{n \times n}, B \in$ $\left(\overline{\mathbb{Z}}_{\max } \llbracket \gamma \rrbracket\right)^{n \times p}$ and $C \in\left(\overline{\mathbb{Z}}_{\max } \llbracket \gamma \rrbracket\right)^{q \times n}$ represent the link between transitions. We refer the reader to [7] for a complete presentation.

The class of uncertain systems, which will be considered, are TEG where the number of tokens and time delays are only known to belong to intervals. Therefore uncertainties can be described by intervals with known lower and upper bounds and the matrices of Equations (15) and (16) are such that $A \in \mathbf{A} \in$ $\mathrm{I}\left(\overline{\mathbb{Z}}_{\max } \llbracket \gamma \rrbracket\right)^{n \times n}, B \in \mathbf{B} \in \mathrm{I}\left(\overline{\mathbb{Z}}_{\max } \llbracket \gamma \rrbracket\right)^{n \times p}$ and $C \in \mathbf{C} \in \mathrm{I}\left(\overline{\mathbb{Z}}_{\max } \llbracket \gamma \rrbracket\right)^{q \times n}$, each entry of matrices $\mathbf{A}, \mathbf{B}, \mathbf{C}$ are intervals with bounds in dioid $\overline{\mathbb{Z}}_{\max } \llbracket \gamma \rrbracket$ with only non-negative exponents and coefficients integer values. By Theorem 4, Equation (15) has the minimum solution $X=A^{*} B U$. Therefore, $Y=C A^{*} B U$ and the transfer function of the system is $H=C A^{*} B \in \mathbf{H}=\mathbf{C A}^{*} \mathbf{B} \in$ $\mathrm{I}\left(\overline{\mathbb{Z}}_{\max } \llbracket \gamma \rrbracket\right)^{q \times p}$, where $\mathbf{H}$ represents the interval in which the transfer function will be lie for all the variations of the parameters .

Figure 1 shows a TEG with 2 inputs and 1 output, which may represent a manufacturing system with 3 machines. Machines $M_{1}$ and $M_{2}$ produce parts assembled on machine $M_{3}$. A token in dotted lines means that the resource can or not to be available to manufacture part. Durations in bracket gives 
the minimal and maximal time spent in the place before contributing to the enabling of the downstream transition.

For instance, machine $M_{2}$ can manufacture 2 or 3 parts and each processing time will last 3 time units. Each manufactured part will spend between 2 and 6 time units in the downstream place before contributing to the enabling of transition $x_{3}$. Entries $\mathbf{A}_{2,2}=\left[3 \gamma^{3}, 3 \gamma^{2}\right]$ and $\mathbf{A}_{3,2}=[2,6]$ describe the intervals in which these parameters evolve. Therefore, we obtain the following interval matrices,

$$
\begin{aligned}
& \mathbf{A}=\left(\begin{array}{ccc}
{\left[2 \gamma^{2}, 5 \gamma\right]} & {[\varepsilon, \varepsilon]} & {[\varepsilon, \varepsilon]} \\
{[\varepsilon, \varepsilon]} & {\left[3 \gamma^{3}, 3 \gamma^{2}\right]} & {[\varepsilon, \varepsilon]} \\
{[3 \gamma, 4 \gamma]} & {[2,6]} & {\left[2 \gamma^{3}, 3 \gamma\right]}
\end{array}\right) \\
& \mathbf{B}=\left(\begin{array}{l}
{[e, e][\varepsilon, \varepsilon]} \\
{[\varepsilon, \varepsilon][e, e]} \\
{[\varepsilon, \varepsilon][\varepsilon, \varepsilon]}
\end{array}\right) \\
& \mathbf{C}=([\varepsilon, \varepsilon][\varepsilon, \varepsilon][e, e]) \text {. }
\end{aligned}
$$

and thanks to theorem 3, the transfer function $H$ belongs to the interval matrix $\mathbf{H}$ given below. It characterizes the whole transfer functions coming from (17):

$$
\mathbf{H}=\mathbf{C A}^{*} \mathbf{B}=\left(\left[3 \gamma\left(2 \gamma^{2}\right)^{*}, 4 \gamma(5 \gamma)^{*}\right]\left[2\left(3 \gamma^{3}\right)^{*}, 6(3 \gamma)^{*}\right]\right)
$$

\section{Robust feedback controller synthesis}

We consider the behavior of a $p$-input $q$-output TEG by a state representation such as (15) and (16), we focus here on output feedback controller synthesis denoted $F$, added between the output $Y$ and the input $U$ of the system (see Figure 2). Therefore the process input verifies $U=V \oplus F Y$, and the output is described by $Y=H(V \oplus F Y)$. According to Theorem 4, the closed-loop transfer relation (depending on $F$ ) is then equal to

$$
Y=(H F)^{*} H V
$$

where $H \in \mathbf{H}$ is the uncertain system transfer.

The objective of the robust feedback synthesis is to compute a controller $F$ which imposes a desired behavior (a specification) to the uncertain system. 
The problem addressed here, consists in computing the greatest interval (in the sense of the order relation $\left.\preceq_{I\left(\overline{\mathbb{Z}}_{\max } \llbracket \gamma \rrbracket\right)}\right)$, denoted $\hat{\mathbf{F}}$, which guarantees that the behavior of the closed loop system is lower than $\mathbf{G}_{r e f} \in \mathrm{I}\left(\overline{\mathbb{Z}}_{\max } \llbracket \gamma \rrbracket\right)^{q \times p}$ (a specification defined as an interval) for all $H \in \mathbf{H}$. Formally the problem consists in computing the upper bound of the following set

$$
\left\{\mathbf{F} \in \mathrm{I}\left(\overline{\mathbb{Z}}_{\max } \llbracket \gamma \rrbracket\right)^{p \times q} \mid(\mathbf{H F})^{*} \mathbf{H} \preceq \mathbf{G}_{r e f}\right\}
$$

Proposition 34 shows that this problem admits a solution for some reference models.

Proposition 34 Let $M_{\mathbf{H}}: \mathrm{I}\left(\overline{\mathbb{Z}}_{\max } \llbracket \gamma \rrbracket\right)^{p \times q} \rightarrow \mathrm{I}\left(\overline{\mathbb{Z}}_{\max } \llbracket \gamma \rrbracket\right)^{q \times p}, \mathbf{F} \mapsto(\mathbf{H F})^{*} \mathbf{H}$ be a mapping. Let us consider the following sets :

$$
\begin{aligned}
& \mathbf{G}_{1}=\left\{\mathbf{G} \in \mathrm{I}\left(\overline{\mathbb{Z}}_{\max } \llbracket \gamma \rrbracket\right)^{q \times p} \mid \exists \mathbf{D} \in \mathrm{I}\left(\overline{\mathbb{Z}}_{\max } \llbracket \gamma \rrbracket\right)^{q \times q} \text { s.t. } \mathbf{G}=\mathbf{D}^{*} \mathbf{H}\right\}, \\
& \mathbf{G}_{2}=\left\{\mathbf{G} \in \mathrm{I}\left(\overline{\mathbb{Z}}_{\max } \llbracket \gamma \rrbracket\right)^{q \times p} \mid \exists \mathbf{D} \in \mathrm{I}\left(\overline{\mathbb{Z}}_{\max } \llbracket \gamma \rrbracket\right)^{p \times p} \text { s.t. } \mathbf{G}=\mathbf{H D}^{*}\right\} .
\end{aligned}
$$

If $\mathbf{G}_{r e f} \in \mathbf{G}_{1} \cup \mathbf{G}_{2}$, there exists a greatest $\mathbf{F}$ such that $M_{\mathbf{H}}(\mathbf{F}) \preceq \mathbf{G}_{r e f}$, given by

$$
\hat{\mathbf{F}}=\underset{\left\{\mathbf{F} \in I\left(\overline{\mathbb{Z}}_{\max } \llbracket \gamma \rrbracket\right)^{p \times q} \mid(\mathbf{H F})^{*} \mathbf{H} \preceq \mathbf{G}_{r e f}\right\}}{\bigoplus} \mathbf{F}=\mathbf{H} \phi \mathbf{G}_{r e f} \phi \mathbf{H}
$$

PROOF. Direct from Proposition 20.

Below, we consider the robust controllers set, denoted $\mathcal{F}$, such that the transfer of the closed loop system be in $\mathbf{G}_{r e f}$ for all $H \in \mathbf{H}$

$$
\mathcal{F}=\left\{F \in \overline{\mathbb{Z}}_{\max } \llbracket \gamma \rrbracket^{p \times q} \mid(\mathbf{H} F)^{*} \mathbf{H} \subset \mathbf{G}_{r e f}\right\}
$$

Corollary 35 If $\mathbf{G}_{\text {ref }} \in \operatorname{Im} M_{\mathbf{H}}$, then $\hat{\mathbf{F}} \subset \mathcal{F}$.

PROOF. If $\mathbf{G}_{r e f} \in \operatorname{Im} M_{\mathbf{H}}$, then $M_{\mathbf{H}}(\hat{\mathbf{F}})=\mathbf{G}_{r e f}$ thanks to Corollary 21, thus $(\mathbf{H} \hat{\mathbf{F}})^{*} \mathbf{H} \subset \mathbf{G}_{r e f}$. Obviously, this is equivalent to $\forall F \in \hat{\mathbf{F}},(\mathbf{H} F)^{*} \mathbf{H} \subset \mathbf{G}_{r e f}$, which leads to the result.

Corollary 35 shows that if $\mathbf{G}_{r e f} \in \operatorname{Im} M_{\mathbf{H}}$ each feedback controller $F \in \hat{\mathbf{F}}$ is also in $\mathcal{F}$. From a practical point of view this means that for all number of tokens and holding time belonging to the given interval the closed loop system 
will be in the specification interval.

Corollary 36 If $\mathbf{G}_{r e f} \in \operatorname{Im} M_{\mathbf{H}}$, then the upper bound of the interval $\hat{\mathbf{F}}$, denoted $\overline{\hat{F}}$, is the upper bound of the set $\mathcal{F}$.

PROOF. Corollary 35 yields $(\mathbf{H} \hat{\mathbf{F}})^{*} \mathbf{H}=\mathbf{G}_{\text {ref }}$, i.e., $\left[(\underline{H \hat{F}})^{*} \underline{H},(\bar{H} \overline{\hat{F}})^{*} \bar{H}\right]=$ $\left[\underline{G}_{r e f}, \bar{G}_{r e f}\right]$. Furthermore $\mathbf{G}_{r e f} \in \operatorname{Im} M_{\mathbf{H}}$ implies that there exists $F$ such that $\bar{G}_{r e f}=(\bar{H} F)^{*} \bar{H}$, i.e., $\bar{G}_{\text {ref }} \in \operatorname{Im} M_{H}$ then thanks to corollary $21 \overline{\hat{F}}=$ $\bar{H} \phi \bar{G}_{r e f} \phi \bar{H}$ is the greatest feedback such that $\bar{G}_{r e f}=(\bar{H} \overline{\hat{F}})^{*} \bar{H}$, thus the greatest feedback in $\mathcal{F}$.

Remark 37 From a computational point of view we have

$$
\begin{aligned}
\hat{\mathbf{F}} & =\mathbf{H} \phi \mathbf{G}_{r e f} \phi \mathbf{H}=[\underline{H}, \bar{H}] \phi\left[\underline{G}_{r e f}, \bar{G}_{r e f}\right] \phi[\underline{H}, \bar{H}]=\left[\underline{H} \phi \underline{G}_{r e f} \wedge \bar{H} \phi \bar{G}_{r e f}, \bar{H} \phi \bar{G}_{r e f}\right] \phi[\underline{H}, \bar{H}] \\
& =\left[\left(\underline{H} \phi \underline{G}_{r e f} \wedge \bar{H} \phi \bar{G}_{r e f}\right) \phi \underline{H} \wedge \bar{H} \phi \bar{G}_{r e f} \phi \bar{H}, \bar{H} \phi \bar{G}_{r e f} \phi \bar{H}\right] \\
& =\left[\underline{H} \phi \underline{G}_{r e f} \phi \underline{H} \wedge \bar{H} \phi \bar{G}_{r e f} \phi \underline{H} \wedge \bar{H} \phi \bar{G}_{r e f} \phi \bar{H}, \bar{H} \phi \bar{G}_{r e f} \phi \bar{H}\right] \text { thanks to (7). }
\end{aligned}
$$

The last equation may be simplified, indeed $\left(\bar{H} \phi \bar{G}_{r e f}\right) \phi \underline{H} \succeq\left(\bar{H} \phi \bar{G}_{r e f}\right) \phi \bar{H}$ thanks to the antitony of mapping a $\phi x$ (i.e., $\left.x_{1} \succeq x_{2} \Rightarrow a \phi x_{1} \preceq a \phi x_{2}\right)$, then $\bar{H} \phi \bar{G}_{r e f} \phi \underline{H \wedge}$ $\bar{H} \phi \bar{G}_{r e f} \phi \bar{H}=\bar{H} \phi \bar{G}_{r e f} \phi \bar{H}$. Therefore

$$
\hat{\mathbf{F}}=\mathbf{H} \phi \mathbf{G}_{r e f} \phi \mathbf{H}=\left[\underline{H} \oint \underline{G}_{r e f} \phi \underline{H} \wedge \bar{H} \phi \bar{G}_{r e f} \phi \bar{H}, \bar{H} \phi \bar{G}_{r e f} \phi \bar{H}\right] .
$$

\section{Example : Output Feedback synthesis}

We describe a complete synthesis of a controller for the uncertain TEG depicted with solid black lines in Fig. 1. The reference model chosen is

$$
\begin{aligned}
G_{r e f} & =\left(\mathbf{H}\left(\begin{array}{l}
\gamma^{2} \\
\gamma^{2}
\end{array}\right)\right)^{*} \mathbf{H} \\
& =\left(\left[3 \gamma \oplus 5 \gamma^{3}(1 \gamma)^{*}, 4 \gamma(5 \gamma)^{*}\right]\left[2 \oplus\left(4 \gamma^{2}\right)(1 \gamma)^{*}, 6 \oplus 9 \gamma \oplus 12 \gamma^{2} \oplus 15 \gamma^{3} \oplus 18 \gamma^{4} \oplus 21 \gamma^{5} \oplus 25 \gamma^{6}(5 \gamma)^{*}\right]\right) .
\end{aligned}
$$

This specification means that not more than two tokens can input in the TEG at the same moment. We refer the reader to $[8, ?]$ for a discussion about reference model choice. We aim to compute the greatest interval of robust controllers which keep the same objective.

According to Proposition 34 and solution (21), the controller is obtained by 
computing $\mathbf{H} \nmid \mathbf{G}_{r e f} \phi \mathbf{H}$. Therefore we obtain

$$
\hat{\mathbf{F}}=\left(\begin{array}{c}
{\left[-3 \gamma^{-1} \oplus-1 \gamma(1 \gamma)^{*},-15 \gamma^{-1}(5 \gamma)^{*}\right]} \\
{\left[-2 \oplus \gamma^{2}(1 \gamma)^{*},-6 \oplus-3 \gamma \oplus \gamma^{2} \oplus 3 \gamma^{3} \oplus 6 \gamma^{4} \oplus 9 \gamma^{5} \oplus 13 \gamma^{6}(5 \gamma)^{*}\right]}
\end{array}\right)
$$

For the realization of that controller it is necessary to choose one feedback in the set $\hat{\mathbf{F}}$. Here we choose the lower bound of this set, i.e.,

$$
\underline{\hat{F}}=\left(-3 \gamma^{-1} \oplus-1 \gamma(1 \gamma)^{*} \quad-2 \oplus \gamma^{2}(1 \gamma)^{*}\right)^{t}
$$

This feedback is not causal because there are negative coefficients in matrix entries meaning negative date for the transition firings (see [2] for a strict definition of causality in dioid). The canonical injection from the set of causal elements of $\overline{\mathbb{Z}}_{\max } \llbracket \gamma \rrbracket$ (denoted $\overline{\mathbb{Z}}_{\max }^{+} \llbracket \gamma \rrbracket$ ) in $\overline{\mathbb{Z}}_{\max } \llbracket \gamma \rrbracket$ is also residuated (see [8] for details). Its residual is denoted $\mathrm{Pr}_{+}$, therefore the greatest causal feedback is

$$
\underline{\hat{F}}_{+}=\operatorname{Pr}_{+}(\underline{\hat{F}})=\left(\begin{array}{c}
\gamma^{2}(1 \gamma)^{*} \\
\gamma^{2}(1 \gamma)^{*}
\end{array}\right)
$$

Figure 1 shows one realization of the controller (bold dotted lines).

Remark 38 The reader can find software tools in order to handle periodic series and solve the illustration (see [19]).

\section{Conclusion}

In this paper we have supposed that the TEG includes some parametric uncertainties in a bounded context. We have given a robust feedback controller synthesis which ensures that the closed-loop system transfer is in a given interval for all feasible values for the parameters. The next step is to extend this work to other control structure such as the one given in [16]. The traditional interval theory is very effective for parameter estimation, it would be interesting to apply the results of this paper to the TEG parameter estimation such as intended in [11]. 


\section{References}

[1] H. Ayhan and M.A. Wortman. Job flow control in assembly operations. IEEE Transactions on Automatic Control, 44(4):864-868, 1999.

[2] F. Baccelli, G. Cohen, G.J. Olsder, and J.P. Quadrat. Synchronization and Linearity : An Algebra for Discrete Event Systems. Wiley and Sons, 1992.

[3] J. Betrema. Topologies sur des espaces ordonnés. R.A.I.R.O. Informatique Théorique, 16(2):165-182, 1982.

[4] T.S. Blyth and M.F. Janowitz. Residuation Theory. Pergamon press, 1972.

[5] J.-Y Le Boudec and P. Thiran. Network Calculus. Springer Verlag, 2002.

[6] G. Cohen. Residuation and applications. Algèbres Max-Plus et applications en informatique et automatique, Ecole de printemps d'informatique théorique, 1998.

[7] G. Cohen, P. Moller, J.P. Quadrat, and M. Viot. Algebraic Tools for the Performance Evaluation of Discrete Event Systems. IEEE Proceedings: Special issue on Discrete Event Systems, 77(1):39-58, January 1989.

[8] B. Cottenceau, L. Hardouin, J.-L. Boimond, and J.-L. Ferrier. Model Reference Control for Timed Event Graphs in Dioid. Automatica, 37:1451-1458, August 2001.

[9] S. Gaubert. Resource Optimization and (min,+) Spectral Theory. IEEE Trans. on Automatic Control, 40(11), November 1995.

[10] M. Gondran and M. Minoux. Graphes, diö̈des et semi-anneaux : Nouveaux modèles et algorithmes. Paris, Tec \& Doc edition, 2001.

[11] L. Jaulin, J.-L. Boimond, and L. Hardouin. Estimation of discrete event systems using interval computation. Reliable Computing, 5:165-173, 1999.

[12] L. Jaulin, M. Kieffer, O. Didrit, and E. Walter. Applied Interval Analysis with Examples in Parameter and State Estimation, Robust Control and Robotics. Springer-Verlag, London, 2001.

[13] G.-L. Litvinov and A.-N. Sobolevskii. Idempotent Interval Analysis and Optimization Problems. Reliable Computing, 7(5), 2001.

[14] G.L. Litvinov, V.P. Maslov, and A.N. Sobolevskii. Idempotent Mathematics and Interval Analysis. (math.NA/9911126), February 2001. Available from http://arXiv.org.

[15] R. Lüders and R. Santos-Mendes. Generalized Multivariable Control of Discrete Event Systems in Dioid. Worshop on Discrete Event Systems, WODES'02, Zaragoza, 2002. 
[16] C.-A. Maia, L. Hardouin, R. Santos-Mendes, and B. Cottenceau. Optimal closed-loop control of timed-event graphs in dioids. To appear in IEEE Transactions on Automatic Control.

[17] E. Menguy, J.-L. Boimond, L. Hardouin, and J.-L. Ferrier. Just in Time Control of Timed Event Graphs: Update of Reference Input, Presence of Uncontrollable Input. IEEE Trans. on Automatic Control, 45(11):2155-2158, November 2000.

[18] Max. Plus. Second Order Theory of (Min,+)-linear Systems and its Application to Discrete Event Systems. In Proceedings of the 30th CDC, Brighton, England, December 1991.

[19] SW2001. Software Tools for Manipulating Periodic Series. http://www.istiaangers.fr $/ \sim$ hardouin/outils.html, http://amadeus.inria.fr/gaubert/papers/max.html., 2001.

[20] W. Wonham. Linear multivariable control : A geometric approach, 3rd edition. Springer Verlag, 1985. 
A Introduction

B Dioids and Residuation

B.1 Residuation theory

B.2 Mapping restriction

C $\quad$ Dioid $\mathcal{C}(\mathcal{D})$

D Dioid and Interval Mathematics

D.1 Interval arithmetic in dioid

D.2 Residuation of interval linear inequations

E Interval arithmetic and Timed Event Graphs

F Robust feedback controller synthesis

G Example : Output Feedback synthesis

H Conclusion 


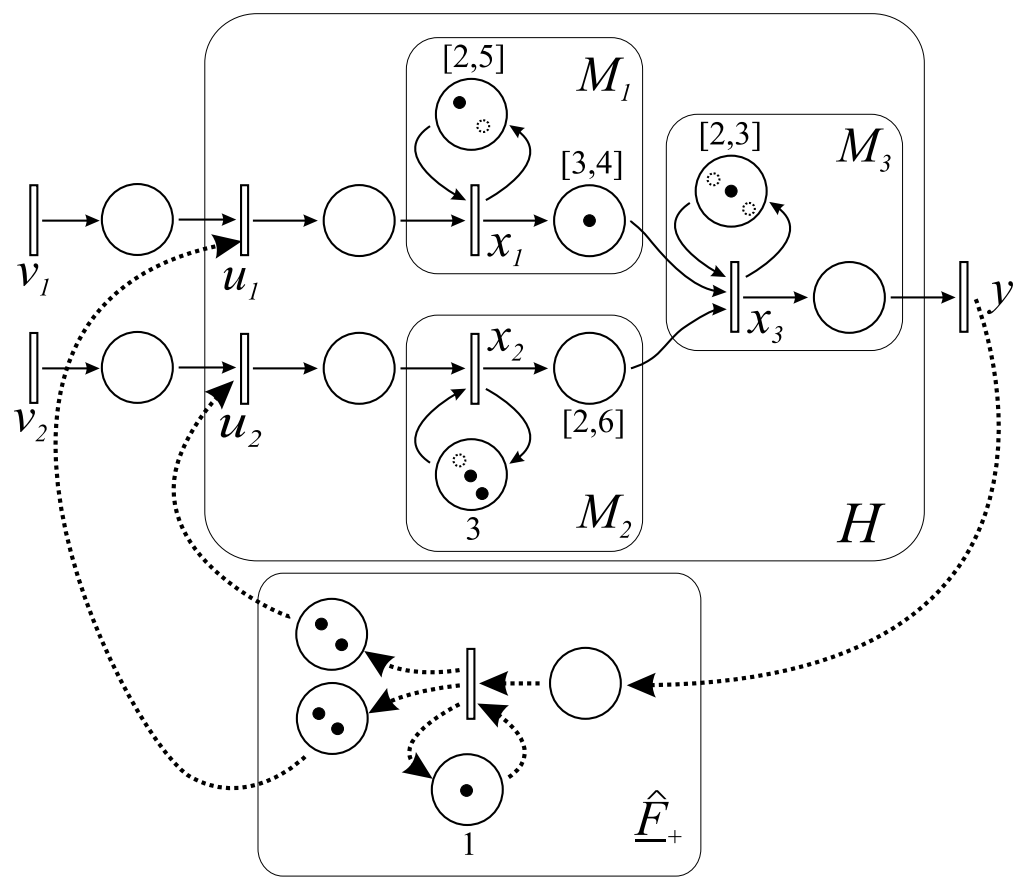

Fig. 1. A uncertain TEG with a controller (bold dotted lines)

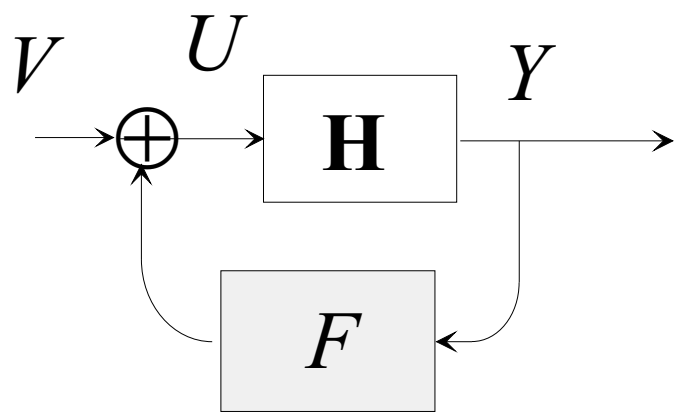

Fig. 2. An uncertain system with a feedback controller 